\title{
Purple Sweet Potato Color Ameliorates Cognition Deficits and Attenuates Oxidative Damage and Inflammation in Aging Mouse Brain Induced by D-Galactose
}

\author{
Qun Shan, ${ }^{1}$ Jun Lu, ${ }^{1}$ Yuanlin Zheng, ${ }^{1,2}$ Jing Li, ${ }^{1}$ Zhong Zhou, ${ }^{1,2}$ Bin Hu, ${ }^{1}$ Zifeng Zhang, \\ Shaohua Fan, ${ }^{1}$ Zhen Mao, ${ }^{1}$ Yong-jian Wang, ${ }^{1}$ and Daifu $\mathrm{Ma}^{2}$ \\ ${ }^{1}$ Key Laboratory for Biotechnology on Medicinal Plants of Province, School of Life Science, Xuzhou Normal University, \\ Xuzhou 221116, China \\ ${ }^{2}$ Department of Potato Genetics, National Sweet Potato Research Institute, Xuzhou 22116, China
}

Correspondence should be addressed to Yuanlin Zheng, ylzheng@xznu.edu.cn

Received 15 February 2009; Revised 24 June 2009; Accepted 7 August 2009

Recommended by R. Lee Mosley

Purple sweet potato color (PSPC), a naturally occurring anthocyanin, has a powerful antioxidant activity in vitro and in vivo. This study explores whether PSPC has the neuroprotective effect on the aging mouse brain induced by D-galactose (D-gal). The mice administrated with PSPC $(100 \mathrm{mg} / \mathrm{kg} \cdot$ day, 4 weeks, from 9th week) via oral gavage showed significantly improved behavior performance in the open field and passive avoidance test compared with D-gal-treated mice ( $500 \mathrm{mg} / \mathrm{kg}$. day, $8 \mathrm{weeks})$. We further investigate the mechanism involved in neuroprotective effects of PSPC on mouse brain. Interestingly, we found, PSPC decreased the expression level of glial fibrillary acidic protein (GFAP), inducible nitric oxide synthase (iNOS), and cyclooxygenase2 (COX-2), inhibited nuclear translocation of nuclear factor-kappaB (NF- $\kappa \mathrm{B})$, increased the activity of copper/zinc superoxide dismutase $(\mathrm{Cu} / \mathrm{Zn}-\mathrm{SOD})$ and catalase (CAT), and reduced the content of malondialdehyde (MDA), respectively. Our data suggested that PSPC attenuated D-gal-induced cognitive impairment partly via enhancing the antioxidant and anti-inflammatory capacity.

Copyright (C) 2009 Qun Shan et al. This is an open access article distributed under the Creative Commons Attribution License, which permits unrestricted use, distribution, and reproduction in any medium, provided the original work is properly cited.

\section{Introduction}

There is considerable evidence that oxidative stress plays an important role during the pathogenesis of age-associated or neurodegenerative disease [1]. Oxidative stress is caused by the imbalance between the reactive oxygen species (ROS) production and ROS elimination in the biological system. And it leads to oxidative damage to cell and tissue paralleled by modifications in the morphology and function, resulting in aging and premature cell death. The aging animal is easily subjected to the alteration of observed behavior such as the reduction of spontaneous behavior, the decline of learning, and memory function. Now a decline in memory and cognitive function is considered to be a consequence of aging and age-related neurodegenerative disorders [2,3]. The age-related impairment in learning and memory may be relieved by antioxidant treatment [4].
One approach to protect against ROS in aging and neurodegenerative disorders is to enhance biological defense activities via antioxidants. Recently, most efforts to prevent and treat neurodegenerative disorders focus on dietary antioxidant components, including vitamins and phenolic compounds [5]. Anthocyanins are a class of naturally polyphenolic compounds that give the intense color to many flowers and fruits in the high plants. They have been reported to show several effectively pharmacological properties such as anti-inflammation, antioxidation $[6,7]$, and antimutagen $[8,9]$. Purple sweet potato (Ipomoea batatas) has been regarded as an abundant source of stable anthocyanins [10] and has been attracted extensive attentions. The previous studies on purple sweet potato color (PSPC) have focused on its stability, structure, and physiological functionality and functional food exploitation [11-13]. However, it is still uncertain whether PSPC 
could improve spontaneous behavior, learning, and memory function, and whether PSPC could have a neuroprotective effect on brain damage induced by the oxidation and inflammation.

D-galactose (D-gal) is a naturally occurring chemical substance in the body; however, at high levels, it can cause the accumulation of ROS, finally resulting in oxidative stress. Recently, D-gal was used to inject mice or rats for pharmacological studies. Evidence has shown that Dgal caused aging-related changes including the increase of ROS and the decrease of antioxidant enzyme activity [14, 15]. D-gal could simultaneously cause the behavioral and neurochemical changes [15-18]. The D-gal-injected mouse has been utilized to be a model for antiaging pharmacology and brain aging studies.

In this study, we investigated the neuroprotective effect of PSPC on impaired explortary behavior, learning, and memory in the D-gal-treated mouse and the underlying neuroprotective mechanism of PSPC on mouse brain.

\section{Material and Methods}

2.1. Animals and Administration. Male Kunming mice (30.23 \pm 5.12 g body weight) were purchased from the Branch of National Breeder Center of Rodents (Shanghai, China). They were housed in a ventilated, temperaturecontrolled $\left(23 \pm 1^{\circ} \mathrm{C}\right)$, and standardized sterile animal room. Eight mice were housed per cage and had free access to food and water under 12 hours light/dark schedule (lights on from 08:30 to 20:30 hour). After acclimatization to the laboratory for one week, group 1 and group 3 were injected (s.c.) with D-gal at dose of $500 \mathrm{mg} /(\mathrm{kg}$.day) (Sigma-Aldrich, MO, USA) [19], and group 2 and group 4 were injected with $0.9 \%$ saline for 8 weeks. Then group 1 and group 4 were administrated by oral gavage with PSPC (Ipomoea batatas; Tsingtao Pengyuan Natural Pigment Research Institute, China; the major components of PSPC by HPLC analysis are cyanidin acyl glucosides and peonidin acyl glucosides (>90\%), and the others are other flavonoids) in distilled water containing $0.1 \%$ Tween 80 at dose of $100 \mathrm{mg} /(\mathrm{kg} . \mathrm{d})$; at the same time other two groups received distilled water containing $0.1 \%$ Tween 80 without PSPC for another four weeks. Before experiment, the mean body weights of each group were examined: control group: $30.08 \pm$ $3.15 \mathrm{~g}$; D-gal group: $31.13 \pm 4.22 \mathrm{~g}$; D-gal + PSPC group: $29.82 \pm 4.71 \mathrm{~g} ;$ PSPC group: $30.16 \pm 2.27 \mathrm{~g}$. After 12 -week administration, the mean body weights of each group were also examined: control group: $38.28 \pm 2.14 \mathrm{~g}$; D-gal group: $39.13 \pm 1.66 \mathrm{~g}$; D-gal + PSPC group: $39.82 \pm 2.59 \mathrm{~g}$; PSPC group: $38.03 \pm 2.33 \mathrm{~g}$. The administration of D-gal and/or PSPC does not significantly influence on the body weight change and the feeding in the mice. The procedures used in the experiment were conducted according to the regulations of the ethics committee of the International Association on the use and care of laboratory animals and were approved by the respective university committees for animal experiments.

\subsection{Behavioral Tests}

2.2.1. Open-Field Testing. The open field has proven useful for evaluation of the effects of drugs on behavior. Locomotor activity and exploratory behavior were examined using a circular arena measuring $50 \mathrm{~cm}$ in diameter and $30 \mathrm{~cm}$ in height with a $40 \mathrm{~W}$ bulb (3000 lux). The floor of the arena was equally divided into 21 units by white lines. Tests were carried out in the animal room from 8:30 to 16:00 as reported previously [20]. Each mouse was placed at the center of the arena. The spontaneous behavioral variables were classified into 4 mutually exclusive categories, including the number of times of rearing, leaning, grooming, and ambulation recoded for 5 minutes.

2.2.2. Passive-Avoidance Testing. The apparatus consisted of an illuminated compartment $(11.5 \mathrm{~cm} \times 9.5 \mathrm{~cm} \times 11 \mathrm{~cm})$ and a dark compartment $(23.5 \mathrm{~cm} \times 9.5 \mathrm{~cm} \times 11 \mathrm{~cm})$. The two compartments were separated by a sliding door, and the dark one contained a metal floor that could deliver footshocks. The illuminated compartment was lit with a $25 \mathrm{~W}$ lamp. The passive avoidance test was performed as described previously [21]. The mouse was firstly placed in the dimly lit room to acclimate the new environment with the apparatus for 0.5 hour before entering the dark compartment. Latency was defined as the interval between the initial opening of the door and the fully entry of the mouse to the dark compartment. The mouse was individually placed in the illuminated compartment facing away from the door. Once the mouse escaped to the illuminated compartment, the door was closed and a mild footshock $(0.3 \mathrm{~mA}, 50 \mathrm{~Hz}, 5$ seconds) was triggered. Immediately the mouse was removed from the apparatus. To carry out the retention trial, the mouse was placed once again in the illuminated compartment 72 hours later. The retention trial followed the method mentioned above in the footshock. The latency (avoidance latency) was recorded. The retention session lasted a maximum ("cutoff") of 300 seconds. The test was conducted in 2 days immediately following the open-field testing.

2.3. Tissue Homogenarate. For biochemical analysis, animals were deeply anesthetized and sacrificed. Brains were promptly dissected and perfused with $50 \mathrm{mM}$ cold phosphate buffer saline solution (PBS, pH7.4). Brains were homogenized in 1/5 (w/v) PBS containing Complete Mini Protease Inhibitor Cocktail tablets (Roche Diagnostics Corporation, Indianapolis, IN, USA) with 10 strokes at $1200 \mathrm{rpm}$ in a Potter homogenizer. Homogenates were divided into two portions. One part was directly centrifuged at $8000 \mathrm{xg}$ for 10 minutes at $4^{\circ} \mathrm{C}$, and the supernatants were collected to measure MDA content. The other part was sonicated four times for 30 seconds with 20 seconds intervals using a VWR Branson Scientific ultrasonicator (VWR Int. Ltd, Merch House Pool, UK). The homogenates were centrifuged at $5000 \mathrm{x}$ g for 10 minutes at $4^{\circ} \mathrm{C}$ to obtain the supernatants for determination of $\mathrm{Cu} / \mathrm{Zn}-\mathrm{SOD}$ and CAT activities.

For western blot analysis, the brain tissues were dissected and homogenized in 1/3 (w/v) Tris-buffered saline (TBS) containing Complete Mini Protease Inhibitor Cocktail 
tablets with 10 strokes at $1200 \mathrm{rpm}$ in a Potter homogenizer. The homogenates were centrifuged at $10000 \mathrm{xg}$ for 10 minutes at $4{ }^{\circ} \mathrm{C}$. And the supernatants were collected and stored at $-70^{\circ} \mathrm{C}$ for the measurement of GFAP, COX-2, and iNOS. Nuclear and cytoplasmic proteins were extracted using an NE-PER kit (Pierce Biotechnology, Inc., Rockford, USA). Nuclear proteins and cytoplasmic proteins were performed by western blot analysis of NF- $\kappa \mathrm{B}$ nuclear translocation. Protein content was determined by using the BCA assay (Pierce Biotechnology, Inc., Rockford, USA).

2.4. Assay of Cu/Zn-SOD and CAT Activity. Cu/Zn-SOD and CAT activities were measured using chemicals purchased from Sigma Chemical Company (USA). Cu/Zn-SOD activity was spectrophotometrically monitored at $550 \mathrm{~nm}$ according to the method described by Sun et al. [22]. SOD activity was showed as $\mathrm{U} / \mathrm{mg}$ protein with reference to the activity of a standard curve of bovine $\mathrm{Cu} / \mathrm{Zn}$-SOD under the same condition.

CAT activity was measured on the basis of Aebi [23]. Briefly, $50 \mu \mathrm{L}$ sample and $0.65 \mathrm{mLof} 50 \mathrm{mM}$ PBS (PH7.0) were added to a quarts cuvette, and $0.3 \mathrm{mLof} 30 \mathrm{mM}$ hydrogen peroxide was used as the substrate. The light absorbance at $240 \mathrm{~nm}\left(25^{\circ} \mathrm{C}\right)$ was determined. Enzymatic activity was expressed as $\mathrm{nM} \mathrm{H}_{2} \mathrm{O}_{2}$ decomposed/min/mg protein.

2.5. Measurement of MDA Level. MDA content was measured using chemicals purchased from Sigma Chemical Company (USA). The level of MDA in brain tissue homogenates was assessed as an index of lipid peroxidation by the method of Mihara and Uchiyama [24]. $500 \mu \mathrm{L}$ homogenate was mixed with 3 mLof $\mathrm{H}_{3} \mathrm{PO}_{4}$ solution ( $1 \%$, $\mathrm{v} / \mathrm{v})$ followed by addition of $1 \mathrm{mLof}$ thiobarbituric acid solution $(0.67 \%, \mathrm{w} / \mathrm{v})$. Then the mixture was heated in a water bath $\left(95^{\circ} \mathrm{C}\right)$ for 45 minutes. The colored complex was extracted into $n$-butanol and cooled to the room temperature. The absorption at $532 \mathrm{~nm}$ was measured using tetramethoxypropane as standard. MDA level was expressed as nmol per mg of protein.

2.6. Western Blot Analysis of GFAP, COX-2, iNOS, and NF$\kappa B$. Sample buffer $(0.1 \mathrm{M}$ Tris- $\mathrm{HCl}, 20 \%$ glycerol, $4 \%$ SDS, and $0.01 \%$ bromophenol blue) was added to aliquot of the supernatant fraction from the lysates. Samples were boiled for 5 minutes. Aliquots of $80 \mu \mathrm{g}$ protein were subjected to $10 \%$ SDS-PAGE. The separated proteins were transferred to PVDF membrane (Roche Diagnostics Corporation, Indianapolis, IN, USA) for 1 hour at $350 \mathrm{~mA}$. The membrane was preblocked with $5 \%$ nonfat milk or $5 \% \mathrm{w} / \mathrm{v}$ BSA in TBS containing $0.1 \%$ Tween 20 , incubated with the primary antibody at $4^{\circ} \mathrm{C}$ with gentle shaking, overnight. Each membrane was washed 3 times for 5 minutes and incubated with the secondary horseradish peroxidase-linked antibody (Santa Cruz Biotechnology, CA and Cell Signaling Technology, Beverly, MA, resp.). Quantitation of detected bands was performed with the Scion Image analysis software (Scion Corp., Frederick, MD, USA). Each density was normalized by corresponding $\beta$-actin ( $40 \mu \mathrm{g}$ sample) or $\beta$-tubulin ( $40 \mu \mathrm{g}$ sample) as an internal control. We standardized the density of control group for relative comparison as 1.0 to compare other group.

2.7. Statistical Analysis. All statistical tests were performed with the statistical program SPSS 11.5. One-way analysis of variance (ANOVA) was applied with Newman-keuls or Tukey's HSD posthoc comparisons. The data were expressed as means \pm SEM. Statistical significance was set at $P<.05$.

\section{Results}

\subsection{Effect of PSPC on the Behavior of D-Gal-Treated Mouse}

3.1.1. Open-Field. Open-field behaviors were observed to identify the behavioral difference in response to a novel environment. The behaviors included exploratory, adaptive, and locomotor abilities of animals expressed by line crossing, rearing/leaning and grooming. The data presented in Figure 1 indicated that, in comparison to the control group with injection of saline $(0.9 \%)$, the behavior activities of mice group subcutaneously injected by using $\mathrm{D}$-gal (D-gal model) cut down on the activities of line crossing $(F(3,28)=6.210$, $P<.01)$, rearing/leaning $(F(3,28)=6.292, P<.01)$, and grooming $(F(3,28)=7.484, P<.001)$. This result showed that the impairment of the exploration activities appeared in the mice treated with D-gal. When D-gal-induced mice were received $100 \mathrm{mg} /(\mathrm{kg} . \mathrm{d})$ of PSPC, their activities of crossing $(P<.001)$, rearing/leaning $(P<.001)$, and grooming $(P<$ $.001)$ were significantly increased. However, there was no statistically significant between the control group and the study group only received PSPC.

3.1.2. Passive-Avoidance Testing. In the initial trial, the passive-avoidance latency showed no difference among the four groups $(F(3,28)=0.622, P>.05)$ (See Figure 2). However, the latency in the 72 hours retention trial was significantly decreased $(F(3,28)=6.476, P<.01)$ in the Dgal-treated mice, as compared with the control. In contrast to the D-gal model, the latency of the D-gal-treated mice fed with $100 \mathrm{mg} /(\mathrm{kg} . \mathrm{d})$ PSPC for 4 weeks was significantly increased $(P<.05)$. However, no significant difference was found between the study group only treated with PSPC and the control group.

3.2. Effect of PSPC on the Cu/Zn-SOD and CAT Activity. $\mathrm{Cu} / \mathrm{Zn}-\mathrm{SOD}$ and CAT are two kinds of important enzymes in the antioxidant defense system. $\mathrm{Cu} / \mathrm{Zn}$-SOD can catalyze the conversion of $\mathrm{O}_{2}^{-}$into $\mathrm{H}_{2} \mathrm{O}_{2}$; furthermore, CAT converts $\mathrm{H}_{2} \mathrm{O}_{2}$ to $\mathrm{H}_{2 \mathrm{O}}$ and $\mathrm{O}_{2}$. To determine whether PSPC could ameliorate oxidative damage in the brain of $\mathrm{D}$-gal-treated mouse, we measured the activities of antioxidant enzymes $\mathrm{Cu} / \mathrm{Zn}-\mathrm{SOD}$ and CAT. Figure 3 showed the activities of $\mathrm{Cu} / \mathrm{Zn}-\mathrm{SOD}$ and CAT in mouse brain. We found from the two figures, compared with control, that D-gal treatment significantly decreased $\mathrm{Cu} / \mathrm{Zn}-\mathrm{SOD}(P<.001$, Figure $3(\mathrm{a}))$ and CAT $(P<.05$, Figure $3(\mathrm{~b}))$ activities, while PSPC could reverse the above changes to the normal level. And there was 


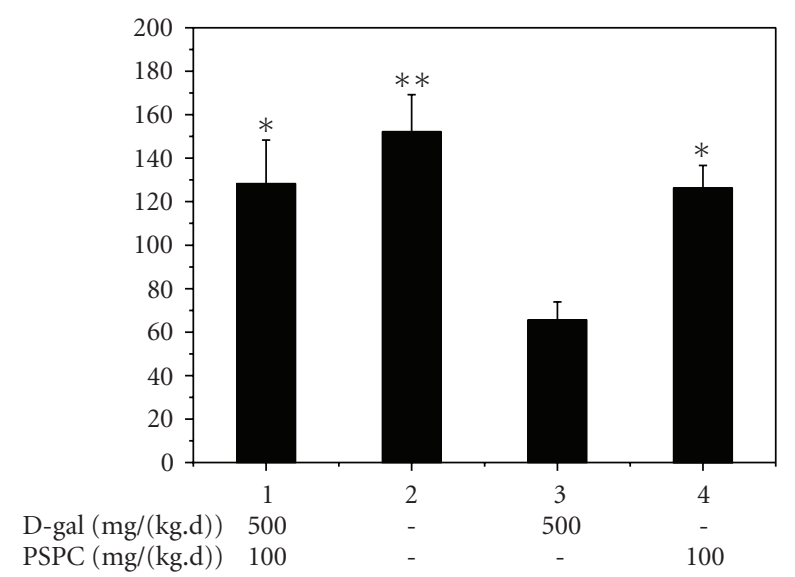

Crossing

(a)

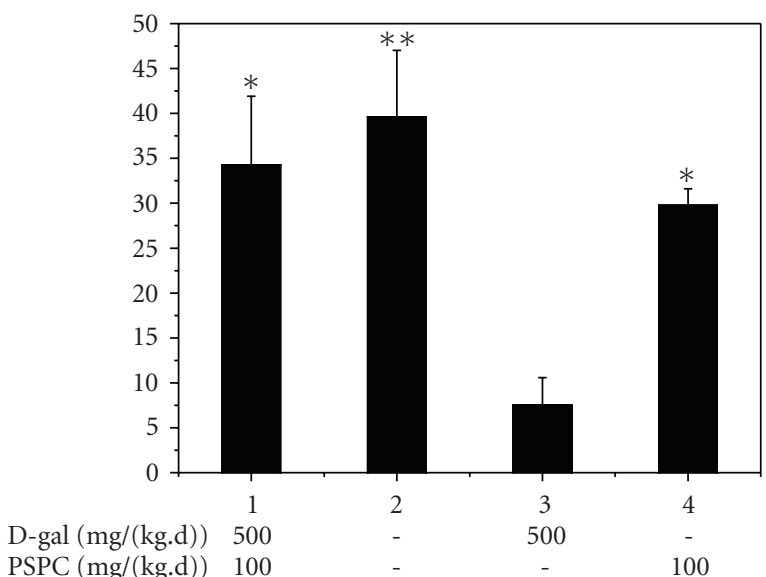

Rearing/leaning

(b)

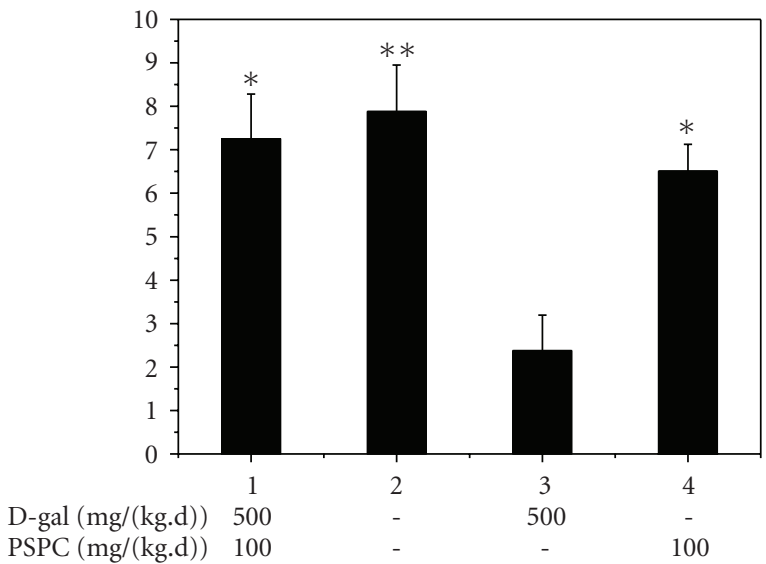

Grooming

(c)

FIgURE 1: Effects of PSPC on open-field behaviors of mice as measured by (a) the number of grid crossing, (b) rearing and leaning, and (c) grooming. All values are expressed as means \pm SEM $(n=8) .{ }^{*} P<.05,{ }^{* *} P<.01,{ }^{* * *} P<.001$ versus $\mathrm{D}$-gal model. Scale bars: $100 \mu \mathrm{M}$.

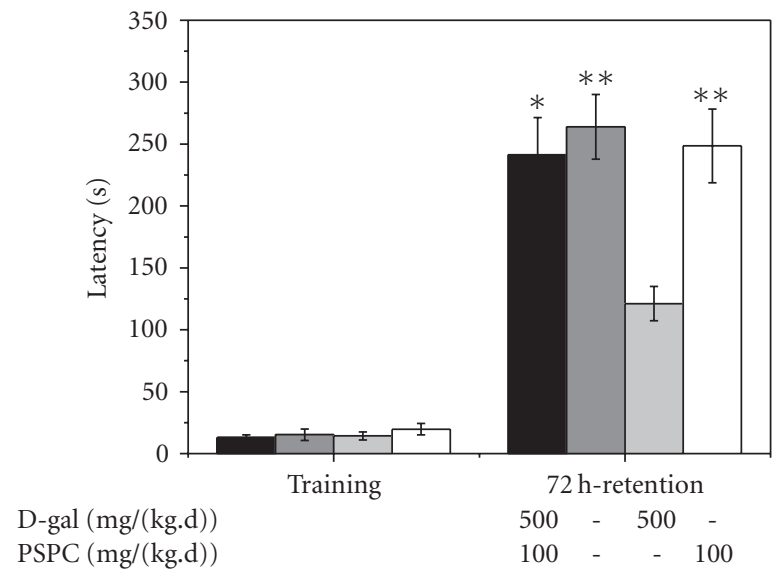

FIGURE 2: Performance in the passive avoidance test. All values are expressed as means \pm SEM $(n=8)$. ${ }^{*} P<.05,{ }^{* *} P<.01$, ${ }^{* * *} P<$ .001 versus D-gal model. Scale bars: $100 \mu \mathrm{M}$.

no significant difference between PSPC group and control group.

3.3. Effect of PSPC on the MDA Content. MDA is a highly reactive three carbon dialdehyde produced as a byproduct of polyunsaturated fatty acid peroxidation and arachidonic acid metabolism and readily combines with several functional groups on molecules including proteins, lipoproteins, and DNA. Therefore, MDA is often measured as a major marker of lipid peroxidation in the aging tissue [25]. As shown in Figure 4, MDA level in D-gal-treated group was higher than that in the control group $(P<.001)$. PSPC depressed MDA enhancement in mouse brain (D-gal/PSPC versus Dgal model, $P<.01)$. While compared with control group, both D-gal/PSPC group and PSPC group did not show significant change.

3.4. Effect of PSPC on the Expression of iNOS, COX-2, and Nuclear Translocation of NF- $\kappa B$. A marker of CNS inflammation is the activation of microglia that produces neurotoxic factor nitric oxide (NO). Generally, the inducible NOS could generate NO, which played an important role in inflammatory response [26]. Our Western blot analysis revealed that D-gal upregulated the expression of iNOS protein $(P<.01$ versus control group), and its level was significantly decreased after PSPC administration. Compared with control group, there was no significant difference in Dgal/PSPC group and PSPC group (Figure 5(a)).

COX-2 is a rate-limiting enzyme in converting arachidonic acid to prostaglandins. The increasing introduction of prostaglandins in the body can lead to the inflammatory response. We found from Figure 5(b) that D-gal-treatment caused an increase in COX-2 expression in mouse brain $(P<$ .001 versus control). When $\mathrm{D}$-gal-treated mice were fed with PSPC, COX-2 level dramatically declined $(P<.001$ versus $\mathrm{D}$-gal model), while PSPC treatment alone did not affect the basal COX-2 expression.

COX-2 promoter contains the binding site for transcription factor of NF- $\kappa \mathrm{B}$ [27]. NF- $\kappa \mathrm{B}$ translocation means its 


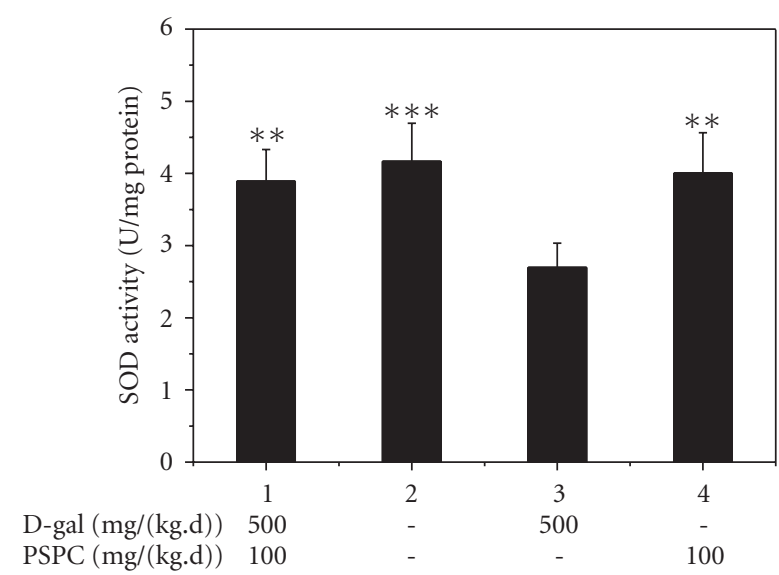

(a)

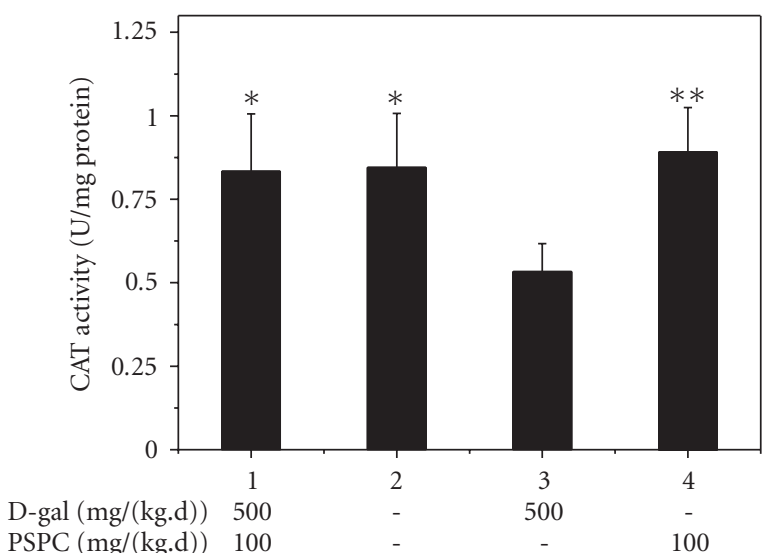

(b)

Figure 3: Pharmacological column for the effect of PSPC on the activity of $\mathrm{Cu} / \mathrm{Zn}-\mathrm{SOD}$ (a) and CAT (b). Each value is the means $\pm \operatorname{SEM}(n=5) .{ }^{*} P<.05,{ }^{* *} P<.001,{ }^{* * *} P<.001$ versus $\mathrm{D}$-gal model.

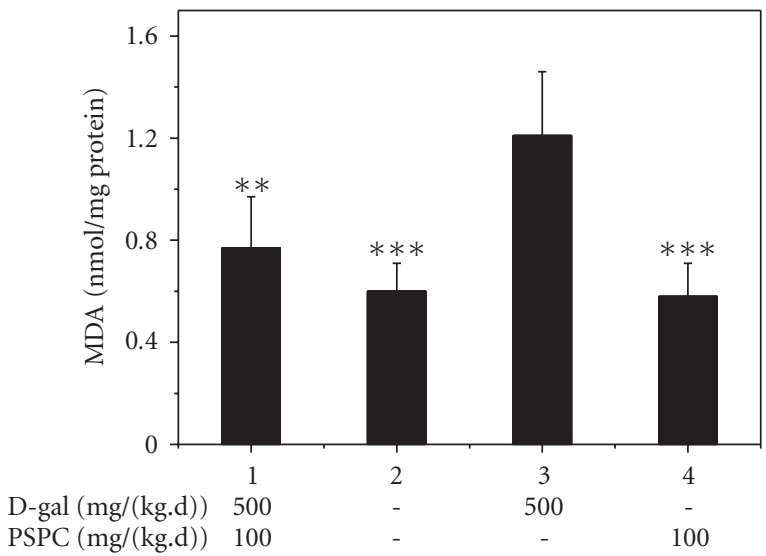

Figure 4: Pharmacological column for effect of PSPC on MDA content. Each value is the means $\pm \operatorname{SEM}(n=5) .{ }^{*} P<.05,{ }^{* *} P<$ .01 , *** $P<.001$ versus $\mathrm{D}$-gal model. activation from the cytosol to the nucleus. At the same time we investigated NF- $\kappa$ B level in the nucleus. The results showed that PSPC markedly attenuated the activity of NF$\kappa \mathrm{B}$ induced by $\mathrm{D}$-gal (D-gal/PSPC versus $\mathrm{D}$-gal model, $P<$ .01 , Figure $5(\mathrm{c}))$. However, there is no statistical variation between PSPC group and control group.

3.5. Effect of PSPC on the Expression of GFAP Protein. GFAP was an intermediate filament that mediates the extension of astrocyte processes, and it has been considered as a reliable marker to document age-related increase in brain of rodents and humans subjects [28-31]. We measured the GFAP expression in mouse brain. As shown in Figure 6, there was a sharp contrast to control group in D-gal-treated group, where twofold amount of GFAP was detected $(P<$ $.01)$. Compared with control group, there was no significant variation in D-gal/PSPC group and PSPC group. Thus, the supplement of PSPC appears to attenuate the production of GFAP protein induced by $\mathrm{D}$-gal.

\section{Discussion}

Aging represents a fundamental biological phenomenon; it can be considered as an inevitable progressive process related to the accumulation of oxidative damage. During normal aging, the brain undergoes morphologic and functional changes resulting in the observed behavioral retrogression such as declines in motor and cognitive performance. These declines are augmented by neurodegenerative diseases including amyotrophic lateral sclerosis (ALS), Alzheimer's disease (AD), and Parkinson's disease (PD) [32]. Currently it has been an encouraging challenge for genetic biology to discover an effective therapy for aging. Therapies in aging may be aimed at reversing or decelerating age-related modifications once they have occurred at various points in the life span [33]. D-gal is a reducing sugar and can be metabolized at normal concentration. However, at high levels, it can be converted into aldose and hydroperoxide under the catalysis of galactose oxidase, resulting in the generation of a superoxide anion and oxygen-derived free radicals [10]. D-gal also reacted readily with the free amines of amino acids in proteins and peptides in vivo to form advanced glycation endproducts (AGEs) [10]. Evidence showed that AGEs could remarkably cause the accumulation of ROS and also trigger neuronal inflammatory through activation of NF- $\kappa \mathrm{B}$ pathway, which induced the behavioral impairment of learning and memory and decreased antioxidant enzyme activities in the brain. By this token, ROS, AGEs, and inflammatory response induced by $\mathrm{D}$-gal contributed to the neurotoxic effect $[10,15,20,34]$. These changes are considerably similar to the normal aging process. So the mouse treated with D-gal has been served as the aging model and has been utilized to investigate the mechanism related to the aging. Flavonoids are unique phytochemicals and have powerful antioxidants. They appear potential in antiaging and antiinflammation properties $[35,36]$. Purple sweet potato color is one of the major flavonoids and has displayed some physiological effects and food exploitation. In this study, 


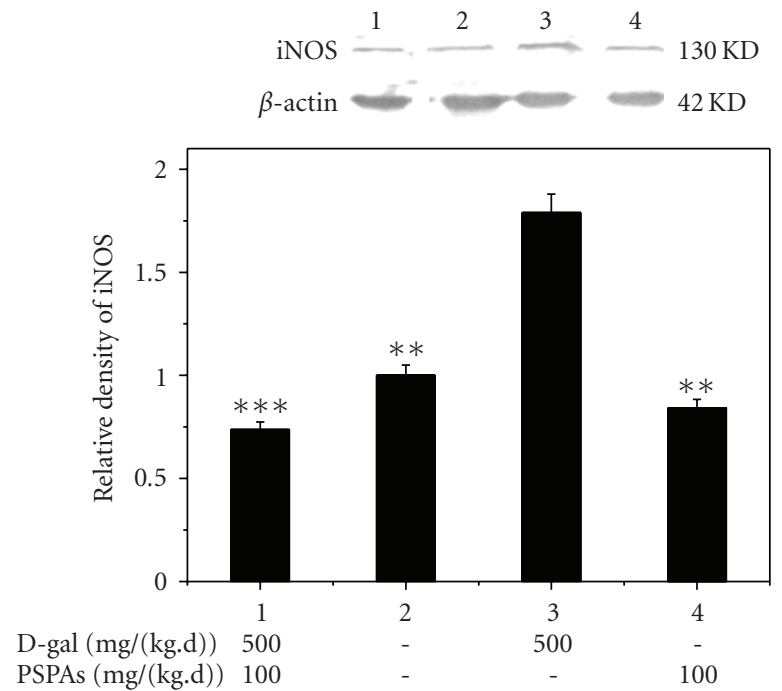

(a)
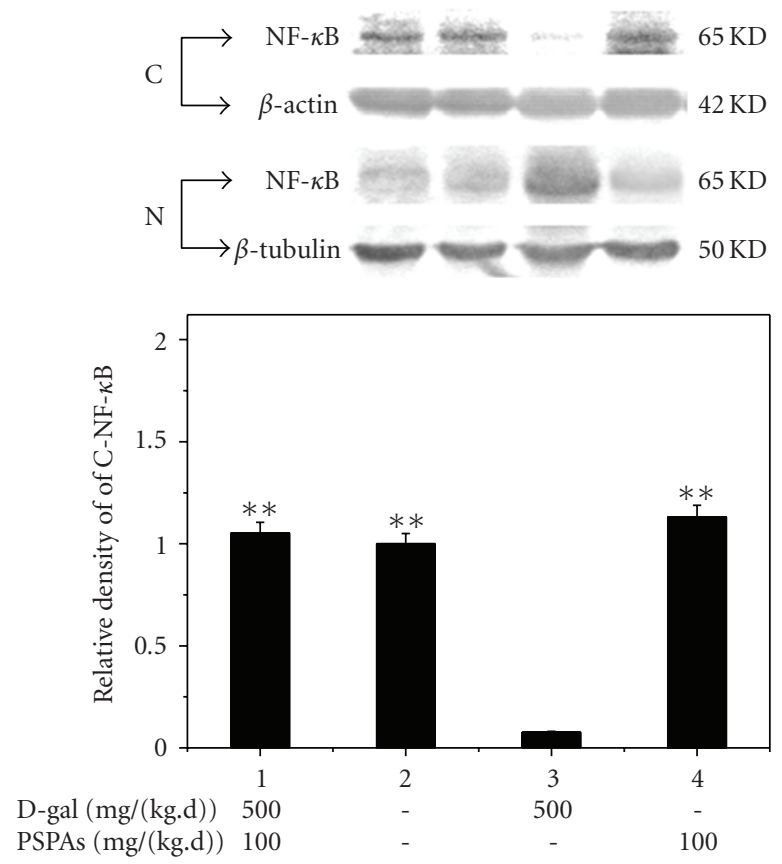

(c)

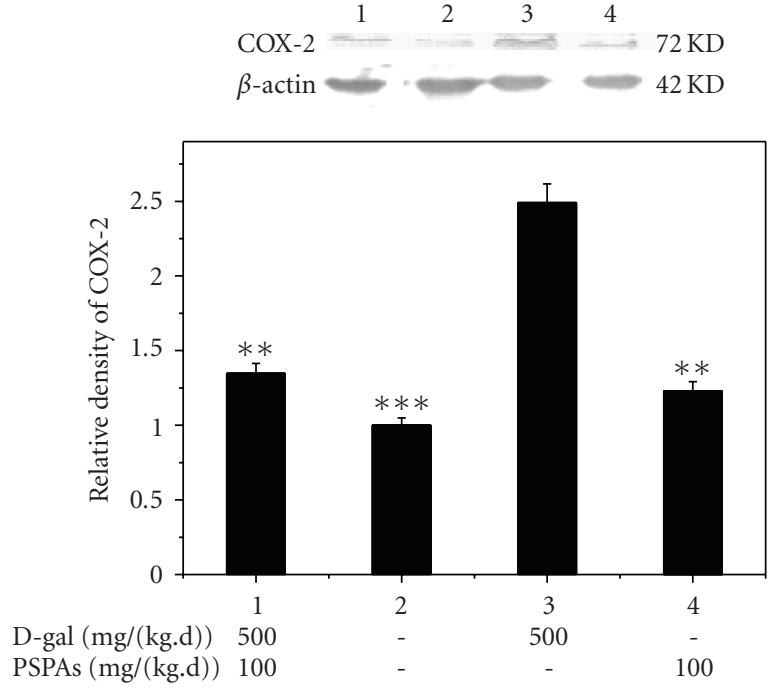

(b)

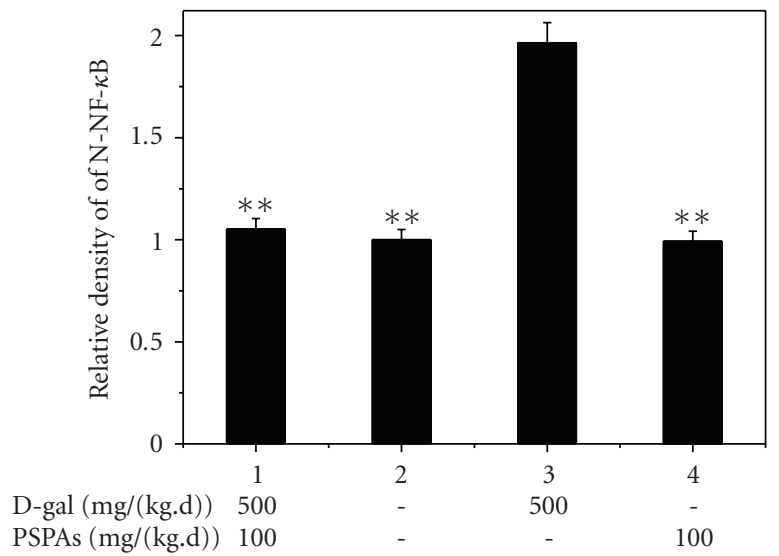

(d)

Figure 5: Western blot analysis of the expression level of representative protein in mouse brain $(n=3)$. (a) iNOS, (b) COX-2, (c) NF- $\kappa$ B: cytoplasmic (C) proteins were isolated and used for western blot analysis, (d) NF- $\kappa$ B: Nuclear $(\mathrm{N})$ proteins were isolated and used for western blot analysis. Data are averages from three independent experiments. Each value is the means \pm SEM $* P<.05,{ }^{* *} P<.01, * * * P<.001$ versus D-gal model.

PSPC is mostly composed of cyanidin acyl glucosides and peonidin acyl glucosides (peonidin 3-O-(6-O-(E)-caffeoyl2-O- $\beta$-D-glucopyranosyl- $\beta$-D-glucopyranoside) $-5-\mathrm{O}-\beta$-Dglucoside, peonidin 3-O-(2-O-(6-O-(E)-caffeoyl- $\beta$-D-glu copyranosyl)-6-O-(E)-caffeoyl- $\beta$-D-glucopyranoside)-5$\mathrm{O}-\beta$-D-glucopyranoside, peonidin $3-\mathrm{O}-(2-\mathrm{O}-(6-\mathrm{O}-(\mathrm{E})-$ feruloyl- $\beta$-D-glucopyranosyl)-6-O-(E)-caffeoyl- $\beta$-D-gluco pyranoside)-5-O- $\beta$-Dglucopyranoside, cyanidin 3-O-(6-Op-coumaroyl)- $\beta$-D-glucopyranoside) by HPLC analysis [37].
Evidence showed that cyanidin acyl glucosides and peonidin acyl glucosides had higher antioxidant and antiinflammatory capacity [38]. The acylated cyanidin and peonidin as the major components of PSPC played an important role in pharmacological effect of PSPC. Our results also demonstrated that the PSPC is an effective antioxidant and anti-inflammatory agent. It could alleviate or reverse the $\mathrm{D}$-gal-caused neurotoxicity by regulating the ROS levels in mouse brain. 

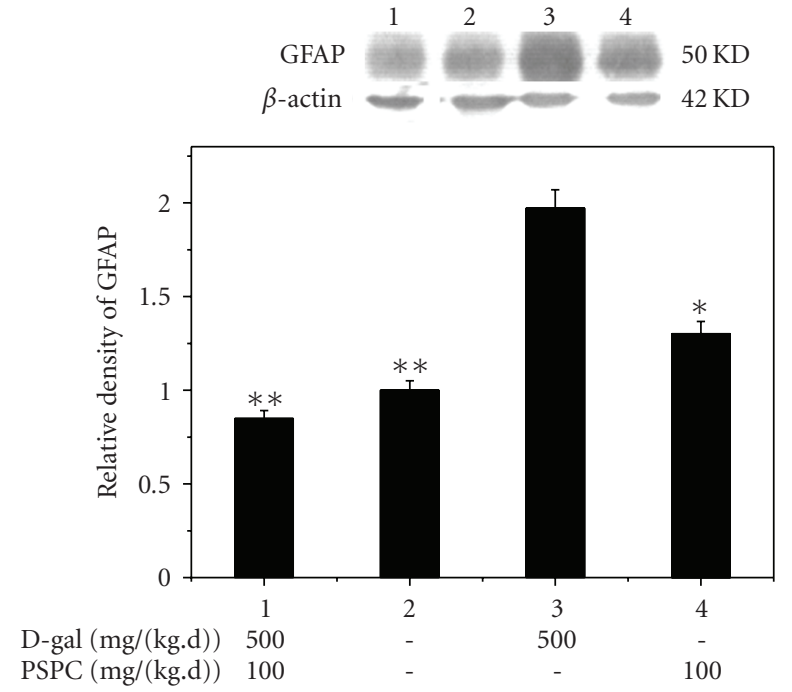

Figure 6: Western blot analysis of the expression level of GFAP protein in mouse brain $(n=3)$. Data are averages from three independent experiments. Each value is the means \pm SEM ${ }^{*} P<$ $.05,{ }^{* *} P<.01,{ }^{* * *} P<.001$ versus $\mathrm{D}$-gal model.

In our behavioral tests, we found that $\mathrm{D}$-gal could remarkably lead to the impairment of learning and memory in mice. However, both open-field and passive avoidance task suggested that there appeared a novel effect of PSPC on the improvement of brain functions during aging. On one hand, PSPC significantly inhibited the behavioral retrogression, such as rearing/leaning, grooming, and crossing, induced by D-gal. PSPC could also reverse the impairment of learning and memory in D-gal-treated mice.

To further identify the molecular mechanism associated with the recovery capability of the aging brain by PSPC treatment following D-gal-induced injury, we utilized the western blot analysis and biochemistry evaluation to study the relative expression of representative protein or enzyme or metabolic product-associated aging in mouse brain. Brain aging is accompanied by behavioral deficits, including cognitive and motor performances. These deficits are probably induced by oxidative damage and inflammation [32, 39]. The excessive ROS produced would speed up the breakdown of biomacromolecules. thus the body could be subjected to the oxidative damage. However antioxidant enzymes can greatly attenuate oxidative damage, providing support for the free radical theory of aging [40]. D-gal could remarkably cause the decrease of antioxidant enzyme activity such as $\mathrm{Cu} / \mathrm{Zn}-\mathrm{SOD}$ and CAT (Figure 3) and the accumulation of ROS, whereas PSPC could restore the activity of $\mathrm{Cu} / \mathrm{Zn}-\mathrm{SOD}$ and CAT and significantly enhanced the antioxidant capacity of the body. The increased MDA content is an important indicator of oxidative damage in brain [40]. We also found that PSPC decreased the generation of MDA induced by Dgal and reduced the production of the free radical. Therefore, PSPC retarded the aging rate induced by D-gal through the alleviation of oxidative damage.

Oxidative damage is one of the main factors of brain aging. The studies also suggest that brain aging is associ- ated with the neuroinflammation. Evidence indicated that oxidative stress and AGEs induced by D-gal both caused neuroinflammation $[41,42]$. Neuroinflammation had been connected to the activation of inflammatory factors iNOS and COX-2, the upregulation of GFAP expression, and the promotion of nuclear translocation of NF- $\kappa \mathrm{B}$ [4347]. Figures 5(a)-5(c) and Figure 6 indicated that D-galtreatment caused inflammatory response, in which iNOS, COX-2, and GFAP levels were increased, and NF- $\kappa$ B activity was also upregulated. It was remarkable that PSPC could reverse the injurious effect of D-gal. This kind of inhibitory function from PSPC is likely to be due to the impactful effect of the anthocyanins on the inflammation. Pergola et al. found that cyanidin from the blackberry extract effectively brought down the expression of iNOS protein induced by proinflammatory stimuli bacterial lipopolysaccharide (LPS) [48]. The other report revealed that cyanidin inhibited the expression of COX-2 and NF- $\kappa$ B induced by LPS as well [6]. Our results indicated that PSPC not only downregulated the expression of COX-2 and iNOS protein but also inhibited nuclear translocation of NF- $\kappa \mathrm{B}$ which was associated with the decreased AGEs levels (data not shown). So PSPC significantly attenuated the progressive inflammation induced by $\mathrm{D}$-gal in mouse brain. Actually, NF- $\kappa \mathrm{B}$ was a transcriptional regulator that could control the expression of iNOS gene and COX-2 gene in modulating inflammation and oxidative stress associated with brain aging $[49,50]$. NF- $\kappa$ B signaling system is one of the main stress signaling pathways and/or central mediator in response to oxidant damage [47]. The results suggested that PSPC showed anti-inflammatory effect in the neurotoxin pathway due to its strongly modulating the COX-2 and iNOS expression by reducing NF- $\kappa$ B activation. And PSPC ameliorated D-gal-induced brain impairment partly by inhibiting NF- $\kappa$ B signaling pathway.

Our group previously found that the impairment of memory and the loss of synaptic protein associated with memory formation in D-gal-treated mice may be improved by treatment with PSPC. Similarly the present study further demonstrated that PSPC could attenuate the impairment of memory and the changes of inflammation and aging factors in D-gal-treated mice. The results suggested that PSPC might improve the behavior performance of the mice not only by increasing the level of the synaptic proteins but also by enhancing the antioxidant ability which could resume activity of antioxidant enzymes, attenuate lipid peroxidation and result in inhibiting inflammatory response in mouse brain.

In conclusion, the present study demonstrated that PSPC administration attenuated D-gal-induced agingrelated changes in mouse brain. PSPC increased the activity of $\mathrm{Cu} / \mathrm{Zn}-\mathrm{SOD}$ and CAT, decreased the expression of iNOS and COX-2, downregulated the expression of GFAP and MDA content, and inhibited nuclear translocation of NF- $\kappa$ B. PSPC consequently improved the spontaneous behavior and cognitive performance and enhanced the capacity of the brain inherent antioxidant or anti-inflammation. These findings about the pharmacological efficacy of PSPC would contribute to brain aging research or aging-related diseases research. 


\section{Abbreviations}

$\begin{array}{ll}\text { PSPC: } & \text { Purple sweet potato color } \\ \text { D-gal: } & \text { D-galactose } \\ \text { ROS: } & \text { Reactive oxygen species } \\ \text { GFAP: } & \text { Glial fibrillary acidic protein } \\ \text { iNOS: } & \text { inducible nitric oxide synthase } \\ \text { COX-2: } & \text { Cyclooxygenase-2 } \\ \text { NF- } \kappa \text { B: } & \text { Nuclear factor-kappaB } \\ \text { Cu/Zn-SOD: Copper/Zinc superoxide dismutase } \\ \text { CAT: } & \text { Catalase } \\ \text { MDA: } & \text { Malondialdehyde } \\ \text { PBS: } & \text { Phosphate buffer saline solution } \\ \text { TBS: } & \text { Tris-buffered saline } \\ \text { CNS: } & \text { Central nervous system } \\ \text { NO: } & \text { Nitric oxide } \\ \text { ALS: } & \text { Amyotrophic lateral sclerosis } \\ \text { AD: } & \text { Alzheimer's disease } \\ \text { PD: } & \text { Parkinson's disease } \\ \text { LPS: } & \text { Lipopolysaccharide } \\ \text { AGEs: } & \text { Advanced glycation endproducts. }\end{array}$

\section{Acknowledgments}

This work is supported by the National Natural Science Fundation of China (30950031), the Foundation for " 863 " Project of the Ministry of Science and Technology of China (2004AA241180), by Major Fundamental Research Program of Natural Science Foundation of the Jiangsu Higher Education Institutions of China (07KJA36029), by Fundamental Research Program of Xuzhou Normal University (05XLB12), and by Qing Lan Project of Jiangsu Province, China. The authors Q. Shan and J. Lu contributed equally to this work.

\section{References}

[1] G. Perry, A. Nunomura, K. Hirai, A. Takeda, G. Aliev, and M. A. Smith, "Oxidative damage in Alzheimer's disease: the metabolic dimension," International Journal of Developmental Neuroscience, vol. 18, no. 4-5, pp. 417-421, 2000.

[2] F. I. M. Craik and T. A Salthouse, Handbook of Aging and Cognition, Hillsdale, Erlbaum, NJ, USA, 1992.

[3] G. E. Smith, R. C. Petersen, J. E. Parisi, et al., "Definition, course, and outcome of mild cognitive impairment," Aging, Neuropsychology, and Cognition, vol. 3, no. 2, pp. 141-147, 1996.

[4] D. J. Socci, B. M. Crandall, and G. W. Arendash, "Chronic antioxidant treatment improves the cognitive performance of aged rats," Brain Research, vol. 693, no. 1-2, pp. 88-94, 1995.

[5] C. Ramassamy, "Emerging role of polyphenolic compounds in the treatment of neurodegenerative diseases: a review of their intracellular targets," European Journal of Pharmacology, vol. 545, no. 1, pp. 51-64, 2006.

[6] D.-X. Hou, T. Yanagita, T. Uto, S. Masuzaki, and M. Fujii, "Anthocyanidins inhibit cyclooxygenase-2 expression in LPS-evoked macrophages: structure-activity relationship and molecular mechanisms involved," Biochemical Pharmacology, vol. 70, no. 3, pp. 417-425, 2005.

[7] B. L. Pool-Zobel, A. Bub, N. Schroder, and G. Rechkemmer, "Anthocyanins are potent antioxidants in model systems but do not reduce endogenous oxidative DNA damage in human colon cells," European Journal of Nutrition, vol. 38, no. 5, pp. 227-234, 1999.

[8] T. Tsuda, K. Shiga, K. Ohshima, S. Kawakishi, and T. Osawa, "Inhibition of lipid peroxidation and the active oxygen radical scavenging effect of anthocyanin pigments isolated from Phaseolus vulgaris L.", Biochemical Pharmacology, vol. 52, no. 7, pp. 1033-1039, 1996.

[9] M. Yoshimoto, S. Okuno, M. Yoshinaga, O. Yamakawa, M. Yamaguchi, and J. Yamada, "Antimutagenicity of sweetpotato (Ipomoea batatas) roots," Bioscience, Biotechnology and Biochemistry, vol. 63, no. 3, pp. 537-541, 1999.

[10] D.-m. Wu, J. Lu, Y.-l. Zheng, Z. Zhou, Q. Shan, and D.-F. $\mathrm{Ma}$, "Purple sweet potato color repairs D-galactose-induced spatial learning and memory impairment by regulating the expression of synaptic proteins," Neurobiology of Learning and Memory, vol. 90, no. 1, pp. 19-27, 2008.

[11] G. Fan, Y. Han, Z. Gu, and D. Chen, "Optimizing conditions for anthocyanins extraction from purple sweet potato using response surface methodology (RSM)," Food Science and Technology, vol. 41, no. 1, pp. 155-160, 2008.

[12] M. Kano, T. Takayanagi, K. Harada, K. Makino, and F. Ishikawa, "Antioxidative activity of anthocyanins from purple sweet potato, Ipomoera batatas cultivar Ayamurasaki," Bioscience, Biotechnology and Biochemistry, vol. 69, no. 5, pp. 979988, 2005.

[13] Q. Tian, I. Konczak, and S. J. Schwartz, "Probing anthocyanin profiles in purple sweet potato cell line (Ipomoea batatas L. Cv. Ayamurasaki) by high-performance liquid chromatography and electrospray ionization tandem mass spectrometry," Journal of Agricultural and Food Chemistry, vol. 53, no. 16, pp. 6503-6509, 2005.

[14] A. Liu, Y. Ma, and Z. Zhu, "Protective effect of selenoarginine against oxidative stress in D-galactose-induced aging mice," Bioscience, Biotechnology and Biochemistry, vol. 73, no. 7, pp. 1461-1464, 2009.

[15] W. Wang, S. Li, H.-P. Dong, S. Lv, and Y.-Y. Tang, "Differential impairment of spatial and nonspatial cognition in a mouse model of brain aging," Life Sciences, vol. 85, no. 3-4, pp. 127135, 2009.

[16] X. Cui, P. Zuo, Q. Zhang, et al., "Chronic systemic Dgalactose exposure induces memory loss, neurodegeneration, and oxidative damage in mice: protective effects of R- $\alpha$-lipoic acid," Journal of Neuroscience Research, vol. 84, no. 3, pp. 647654, 2006.

[17] H. Hao, Y. Nancai, F. Lei, et al., "Antiaging effect of purslane herb aqueous extracts and its mechanism of action," Phytotherapy Research, vol. 23, no. 9, pp. i-vii, 2009.

[18] S.-Z. Zhong, Q.-H. Ge, R. Qu, Q. Li, and S.-P. Ma, "Paeonol attenuates neurotoxicity and ameliorates cognitive impairment induced by D-galactose in ICR mice," Journal of the Neurological Sciences, vol. 277, no. 1-2, pp. 58-64, 2009.

[19] S.-C. Ho, J.-H. Liu, and R.-Y. Wu, "Establishment of the mimetic aging effect in mice caused by D-galactose," Biogerontology, vol. 4, no. 1, pp. 15-18, 2003.

[20] J. Lu, Y.-1. Zheng, L. Luo, D.-M. Wu, D.-X. Sun, and Y.-J. Feng, "Quercetin reverses D-galactose induced neurotoxicity in mouse brain," Behavioural Brain Research, vol. 171, no. 2, pp. 251-260, 2006.

[21] C. Castellano, I. B. Introini-Collison, and J. L. McGaugh, "Interaction of $\beta$-endorphin and GABAergic drugs in the regulation of memory storage," Behavioral and Neural Biology, vol. 60, no. 2, pp. 123-128, 1993.

[22] Y. Sun, L. W. Oberley, and Y. Li, "A simple method for clinical assay of superoxide dismutase," Clinical Chemistry, vol. 34, no. 
3, pp. 497-500, 1988.

[23] H. Aebi, "Catalase in vitro," Methods in Enzymology, vol. 105, pp. 121-126, 1984.

[24] M. Mihara and M. Uchiyama, "Determination of malonaldehyde precursor in tissues by thiobarbituric acid test," Analytical Biochemistry, vol. 86, no. 1, pp. 271-278, 1978.

[25] G.-F. Shi, L.-J. An, B. Jiang, S. Guan, and Y.-M. Bao, "Alpinia protocatechuic acid protects against oxidative damage in vitro and reduces oxidative stress in vivo," Neuroscience Letters, vol. 403, no. 3, pp. 206-210, 2006.

[26] K. C. Park, K. C. Chung, Y.-S. Kim, J. Lee, T. H. Joh, and S.-Y. Kim, "Transglutaminase 2 induces nitric oxide synthesis in BV-2 microglia," Biochemical and Biophysical Research Communications, vol. 323, no. 3, pp. 1055-1062, 2004.

[27] J. L. Jimenez, M. A. Iniguez, M. A. Munoz-Fernandez, and M. Fresno, "Effect of phosphodiesterase 4 inhibitors on NFAT-dependent cyclooxygenase-2 expression in human T lymphocytes," Cellular Signalling, vol. 16, no. 12, pp. 1363$1373,2004$.

[28] C. E. Finch, "Neurons, glia, and plasticity in normal brain aging," Neurobiology of Aging, vol. 24, pp. 123-127, 2003.

[29] J. J. Miguel-Hidalgo, S. Nithuairisg, C. Stockmeier, and G. Rajkowska, "Distribution of ICAM-1 immunoreactivity during aging in the human orbitofrontal cortex," Brain, Behavior, and Immunity, vol. 21, no. 1, pp. 100-111, 2007.

[30] T. A. Prolla and M. P. Mattson, "Molecular mechanisms of brain aging and neurodegenerative disorders: lessons from dietary restriction.", Trends in Neurosciences, vol. 24, supplement 11, pp. 21-31, 2001.

[31] C. C. Stichel and H. Luebbert, "Inflammatory processes in the aging mouse brain: participation of dendritic cells and Tcells," Neurobiology of Aging, vol. 28, no. 10, pp. 1507-1521, 2007.

[32] J. A. Joseph, B. Shukitt-Hale, and G. Casadesus, "Reversing the deleterious effects of aging on neuronal communication and behavior: beneficial properties of fruit polyphenolic compounds," The American Journal of Clinical Nutrition, vol. 81, supplement 1, pp. 313-316, 2005.

[33] E. C. Hadley, E. G. Lakatta, M. Morrison-Bogorad, H. R. Warner, and R. J. Hodes, "The future of aging therapies," Cell, vol. 120, no. 4, pp. 557-567, 2005.

[34] C. F. Chen, S. Y. Lang, P. P. Zuo, N. Yang, X. Q. Wang, and C. Xia, "Effects of D-galactose on the expression of hippocampal peripheral-type benzodiazepine receptor and spatial memory performances in rats," Psychoneuroendocrinology, vol. 31, no. 7, pp. 805-811, 2006.

[35] Y.-J. Jeong, Y.-J. Choi, H.-M. Kwon, et al., "Differential inhibition of oxidized LDL-induced apoptosis in human endothelial cells treated with different flavonoids," British Journal of Nutrition, vol. 93, no. 5, pp. 581-591, 2005.

[36] R. Verbeek, E. A. van Tol, and J. M. van Noort, "Oral flavonoids delay recovery from experimental autoimmune encephalomyelitis in SJL mice," Biochemical Pharmacology, vol. 70, no. 2, pp. 220-228, 2005.

[37] F. Qiu, J. Luo, S. Yao, L. Ma, and L. Kong, "Preparative isolation and purification of anthocyanins from purple sweet potato by high-speed counter-current chromatography," Journal of Separation Science, vol. 32, no. 12, pp. 2146-2151, 2009.

[38] G. Chen, K. A. Bower, M. Xu, et al., "Cyanidin-3-glucoside reverses ethanol-induced inhibition of neurite outgrowth: role of glycogen synthase kinase 3 beta," Neurotoxicity Research, vol. 15, no. 4, pp. 321-331, 2009.

[39] J. A. Joseph, R. T. Bartus, D. Clody, et al., "Psychomotor performance in the senescent rodent: reduction of deficits via striatal dopamine receptor up-regulation," Neurobiology of Aging, vol. 4, no. 4, pp. 313-319, 1983.

[40] T. Jayakumar, P. A. Thomas, and P. Geraldine, "Protective effect of an extract of the oyster mushroom, Pleurotus ostreatus, on antioxidants of major organs of aged rats," Experimental Gerontology, vol. 42, no. 3, pp. 183-191, 2007.

[41] M. M. Sousa, S. D. Yan, R. Fernandes, et al., "Familial amyloid polyneuropathy: receptor for advanced glycation end products-dependent triggering of neuronal inflammatory and apoptotic pathways," The Journal of Neuroscience, vol. 21, no. 19, pp. 7576-7586, 2001.

[42] K. C. Wise, S. K. Manna, K. Yamauchi, et al., "Activation of nuclear transcription factor- $\kappa \mathrm{B}$ in mouse brain induced by a simulated microgravity environment," In Vitro Cellular \& Developmental Biology Animal, vol. 41, no. 3-4, pp. 118-123, 2005.

[43] V. Sharma, M. Mishra, S. Ghosh, et al., "Modulation of interleukin-1 $\beta$ mediated inflammatory response in human astrocytes by flavonoids: implications in neuroprotection," Brain Research Bulletin, vol. 73, no. 1-3, pp. 55-63, 2007.

[44] B. Y. Wu and A. C. Yu, "Quercetin inhibits c-fos, heat shock protein, and glial fibrillary acidic protein expression in injured astrocytes," Journal of Neuroscience Research, vol. 62, no. 5, pp. 730-736, 2000.

[45] A. K. Roy, T. Oh, O. Rivera, J. Mubiru, C. S. Song, and B. Chatterjee, "Impacts of transcriptional regulation on aging and senescence," Ageing Research Reviews, vol. 1, no. 3, pp. 367-380, 2002.

[46] I. Rozovsky, C. E. Finch, and T. E. Morgan, "Age-related activation of microglia and astrocytes: in vitro studies show persistent phenotypes of aging, increased proliferation, and resistance to down-regulation," Neurobiology of Aging, vol. 19, no. 1, pp. 97-103, 1998.

[47] S.-O. Yoon, C.-H. Yun, and A.-S. Chung, "Dose effect of oxidative stress on signal transduction in aging," Mechanisms of Ageing and Development, vol. 123, no. 12, pp. 1597-1604, 2002.

[48] C. Pergola, A. Rossi, P. Dugo, S. Cuzzocrea, and L. Sautebin, "Inhibition of nitric oxide biosynthesis by anthocyanin fraction of blackberry extract," Nitric Oxide, vol. 15, no. 1, pp. 3039, 2006.

[49] I. Rahman, J. Marwick, and P. Kirkham, "Redox modulation of chromatin remodeling: impact on histone acetylation and deacetylation, NF- $\kappa \mathrm{B}$ and pro-inflammatory gene expression," Biochemical Pharmacology, vol. 68, no. 6, pp. 1255-1267, 2004.

[50] Y.-J. Surh, J. K. Kundu, H.-K. Na, and J.-S. Lee, "Redoxsensitive transcription factors as prime targets for chemoprevention with anti-inflammatory and antioxidative phytochemicals," Journal of Nutrition, vol. 135, supplement 12, pp. 2993-3001, 2005. 

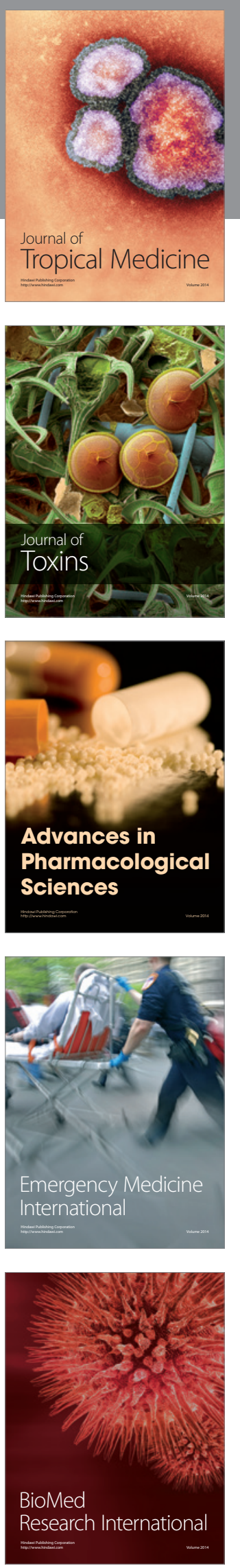
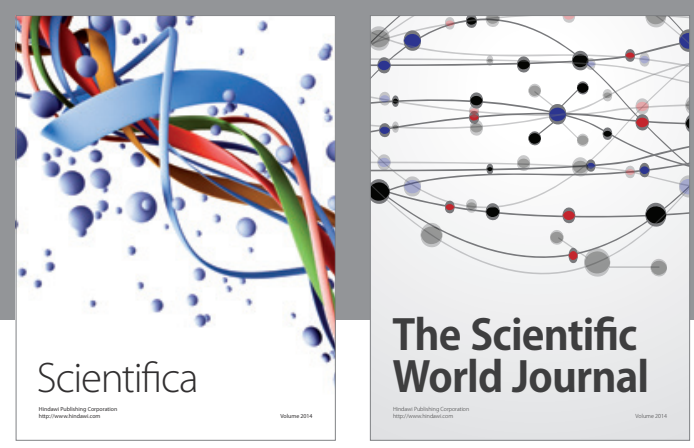

The Scientific World Journal
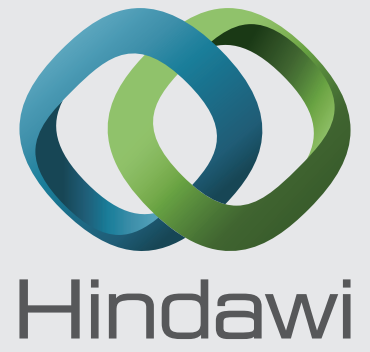

Submit your manuscripts at

http://www.hindawi.com
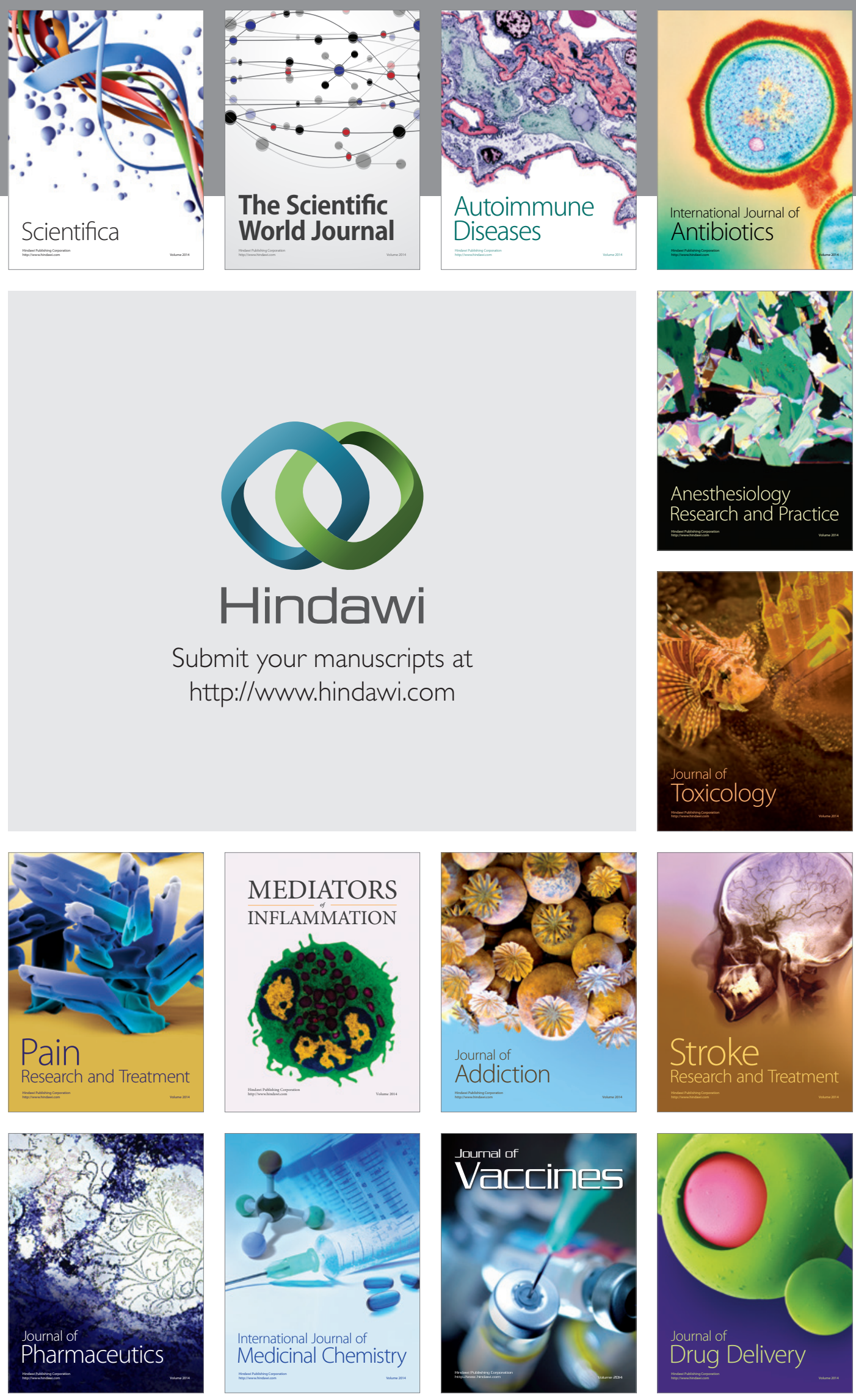